Aus der Obrenklinik des Charité-Krankenhauses in Berlin (dirigirender Arzt: Gel. Med.-Rath Prof. Dr. Trautmann).

\title{
Zur Lehre von den otitischen Hirnabscessen.
}

\author{
Stabsarzt Dr. Riclıard Müller.
}

Ich habe früher bereits über 4 otitische Hirnabscesse berichtet $\left.{ }^{1}\right)$ und bin heute in der Lage, 4 weitere einschlägige Fälle veröffentlichen zu können. Von den frtiher beschriebenen Kranken sind 2 operativ geheilt worden; beide habe ich bis in die jüngste Zeit in Controle behalten, sie sind bis heute am Leben geblieben. Der am 8. Mai 1898 wegen eines Kleinhirnabscesses von mir operirte Patient ist Schmiedegeselle und geht seit Jahr und Tag unbehindert seiner sehweren Arbeit nach, und das Mädchen, bei dem ich am 6. April 1897 einen rechtsseitigen Schläfenlappenabscess entleerte, verdient sich seinen Lebensunterhalt als Näherin; allerdings hat sie zeitweise uber Kopfschmerzen zu klagen und hat auch einige Male, freilich mit grossen Zwisehenräumen, epileptoide Krampfanfälle gehabt.

Bei den neuen Fällen handelt es sich um 2 Schläfen. lappenabscesse, einen rechtsseitigen und einen linksseitigen, und um $2 \mathrm{Kleinhirnabscesse,} \mathrm{ebenfalls} \mathrm{einen} \mathrm{rechtsseitigen}$ und einen linksseitigen. Von diesen 4 Abscessen habe ich 3 operativ entleert, und zwar den linksseitigen Kleinhirnabsoess mit Ausgang in Heilung, den linksseitigen Schläfenlappen- und den rechtsseitigen Kleinhirnabscess, nach vorübergehender Besserung, mit tödtlichem Ausgang - am 19. bezw. 20. Tage nach der Operation - ; der Schläfenlappenabscess rechterseits ist intra vitam nicht diagnosticirt und darum auch nicht operirt worden, er wurde erst bei der Obduction gefunden. Ich will zunächst

1) Drei otitische Schläfenlappenabscesse. Deutsche med. Wochenschrift. 1897. Nr. 53. - Ein operativ geheilter otitischer Kleinhirnabscess. Ebenda. 1898. $\mathrm{Nr}, 49$. 
die Fälle im einzelnen beschreiben, um am Schlusse einige Bemerkungen allgemeiner Natur anzuschliessen.

\section{Schläfenlappenabscess rechts; intra vitam nicht erkannt; Tod.}

Franz S*, 47 Jahre alt, Rollkutscher. Ohren fruber stets gesund. Ende Juni 1898 aus unbekannter Ursache Obrensausen rechts. Paracentese durch den Kassenarzt, darnach fötide Ohreneiterung. Nach 3 Wochen ist diese wieder versiegt, Sausen und Schmerzen im rechten Ohre bleiben. Anfang September Zunahme dieser Beschwerden; der Mann wird daher am 10. September 1898 zur Charité geschickt und hier auf die Ohrenstation aufgenommen

Der Kranke ist einsilbig, geistig nicht ganz klar, daher Aufnabme der Anamnese, die ausser vorgenannten Punkten nichts Wesentliches ergiebt, schwierig. Die einzige Angabe von Belang, die er noch macht, ist die, dass er seit einiger Zeit beim plötzlichen Aufrichten schwindlig werde; uber Kopfschmerzen klagt er nicht. Guter Ernährungszustand, Appetit gut, Stuhl regelmässig, nicht angehalten. Keine Temperatursteigerung. Puls regelmässig, kräftig, 70. Der Gang ist sicher, nicht schwankend. Bei Romberg mässiges Schwanken, aber erst nach längerer Zeit. Die Zunge weicht beim Herausstrecken deutlich und stark nach links ab. Leichte Schwäche des linken Facialis, die sich bis zum 19. September zur vollständigen Lähmung herausbildet. Pupillen gleich, mittelweit, reagiren prompt; Augenbewegungen frei, kein Nystagmus; Augenhintergrund normal, keine Neuritis optica oder gar Stauungspapille. Keine Sprachstörung. Ohren: Keine Spur von Eiterung. Flüstersprache links $2 \mathrm{~m}$, recbts nicht gehört; Weber rechts positiv. Rechts Trommelfell an die Labyrinthwand angeklatscht, die röthlich durchschimmert; oberer Abschnitt durch weissliche Schuppen verdeckt. Links starke Retraction und Trübung. Rechts Abspülen mit ICl 3 -Lösung; es entleeren sich nur einige Epithelschuppen; darnach in der Tiefe immer noch weissliche Massen, die an Cholesteatom erinnern.

In der Nacht vom 18. zum 19. September plötzlich grosse Unruhe; der Mann verlässt das Bett und geht aufgeregt und sicheren Schrittes, aber geistesabwesend im Zimmer auf und ab. Es wird Ausbruch von Delirium potatorum befürchtet und der Mann zur Krampfabtheilung verlegt. Hier stellt sich binnen weniger Stunden Lähmung des linken Armes und Beines ein. Nachmittag völlige Benommenheit, keine Antwort auf Fragen. Patellarreflex fehlt beiderseits. Augenhintergrund immer noch normal. Keine Nackensteifigkeit. Auch auf der Krampfabtheilung wird das Abweichen der Zunge nach links festgestellt. Am 19. September Nachmittag 6 Uhr Tod.

Obductionsbefund: Abcessus cerebri lobi tempor, dextr, $6 \mathrm{~cm}$ lang, $3 \mathrm{~cm}$ breit, $3 \mathrm{~cm}$ hoch; untere Abscesswand $1 \mathrm{~cm}$ dick. Dura auf dem Tegmen tympani durchbrochen und missfarbig.

Der Fall ist leider mangelhaft beobachtet, weil das Fehlen von Ohreneiterung den Gedanken an einen Hirnabscess in uns nicht aufkommen liess; bei der Obduction war ich nicht zugegen, daher fehlen auch hier wichtige Angaben, z. B. liber den Befund am Schläfenbein - ob Caries, Cholesteatom u. s. w. und uber eine etwaige Abscessmembran. Dennoch lassen sich einige interessante und lehrreiche Punkte hervorheben.

Die rasohe Entwickelung des sebr grossen Abscesses innerhalb zweier Monate und trotz seiner Grösse der lange Zeit fast symptomlose Verlauf sind besonders auffallend. Sebwindel beim Aufriehten ist bis zum 10. September fast das einzige Symptom; erst da tritt eine Facialislähmung zu Tage 
und gleichzeitig eine Lähmung des Nervus hypoglossus. Eine solche ist meines Wissens bei einem Schläfenlappenabscess bisher noch nicht beobachtet worden; sie ist auch sonst noch von Interesse insofern, als trotz des Sitzes des ursächlichen centralen Leidens in der rechten Grosshirnhälfte die Zunge beim Herausstrecken doch deutlich nach links, also nach der Seite des gelähmten Nerven hin, abwich (Ueberwiegen des nicht gelähmten M. genioglossus der anderen Seite). Fernwirkung des Abscesses auf die Capsula interna dürfte sowohl für die Facialiswie für die Hypoglossuslähmnng die Erklärung abgeben. Bis zum 18. September, dem Tage vor seinem Tode, war der Mann nicht bettlägerig. Bis dahin fehlte auch das Geftihl des Schwerkrankseins und die sonst doch schon längere Zeit vor dem tödtlichen Ausgange auftretende Schlafsucht; ja, es bestand im Gegentheil eine gewisse Unruhe, die sich noch in der Nacht vor dem Tode zu einem a ufgeregten, an Delirium tremens erinnernden Wesen steigerte. $O b$ die an dem Manne beobachtete Einsilbigkeit und die geistige Unklarheit und Stumpfheit auf den Abscess zurückzuführen oder in der Natur des Kranken an sich - etwa, wie wir annahmen, in Folge von chronischem Alkoholgenuss - begründet war, ist mit positiver Sicherheit nicht zu entscheiden, da wir den Mann früher nicht gekannt haben; doch hat der Verlauf den ersteren Zusammenhang schliesslich wahrseheinlicher gemacht. Wirklich schwere Erscheinungen, Trübung des Bewusstseins und halbseitige Lähmung, traten fast schlaganfallartig erst wenige Stunden vor dem Tode auf, und die gewöhnlichsten Erscheinungen von erhöhtem Hirndruek - Neuritis optica bezw. Staunngspapille, Pulsverlangsamung und Kopfschmerzen - kamen uberhaupt nieht zur Beobachtung. Speciell auf letzteren Punkt werde ich am Schlusse noch zurdickkommen. Bemerkenswerth ist auch, dass die Ohreneiterung sehon lange vorher zum Stillstand gekommen war, ein seltenes Vorkommniss und in unserem Falle besonders verhängnissvoll, weil wir deshalb nicht auf die richtige Diagnose gekommen sind. Ueberhaupt scheint mir der rechtsseitige Schläfenlappenabscess von allen Hirnabseessen derjenige zu sein, dessen Diagnose die grössten Schwierigkeiten macht.

\section{Schläfenlappenabscess links; operativ entleert; Tod am 19. Tage nach der Operation.}

Hermann M , 16 Jahre alt, Buidnerssohn. Kommt am 16. September 1898 zum ersten Miale auf unsere Klinik; schwerkrank; wird sofort aufge- 
nommen. Vor 3 Jahren, nach Angabe der Mutter, Diphtherie; seitdem Ohrenlaufen links. Vor 8 Wochen aus unbekannter Ursache plötzlich Zunabme der Eiterung, der Junge wird immer kränker, desbalb bringt ihn seine Mutter nach Berlin.

17. September. M. ist schwerkrank, liegt apathisch im Bett, ist benommen und antwortet auf Fragen nur langsam, meist erst auf wiederholtes und eindringliches Befragen, doch sind seine Antworten, wenn er sie einmal giebt, richtig und logisch. Klagt von selbst nur über beftige Kopfsehmerzen, die er bei aller seiner Benommenheit stets und ausschliesslich nach der linken Kopfhälfte verlegt. Auf Befragen klagt er auch aber zeitweiligen Schwindel; kann sich im Bett allein aufrichten, doch geht das langsam vor sich, und dabei tritt Schwindelgefühl auf; es sei ihm, als drehe sich alles um ihn herum. Beim Sitzen B rechneigung, aber kein Erbrecben. Nirgends Lähmungserscheinungen. Stuhl nicht angehalten. Gang taumelnd, gespreizt; bei Romberg starkes Schwanken. Kopf nach allen Richtungen hin frei beweglich, wird aber beim Gehen vorsichtig unbewegt gehalten. Kniephänomen + . Augenbewegungen frei, kein Nystagmus, Pupillen gleich weit, reagiren prompt. Augenhintergrund rechts normal, links Schlängelung der Venen ohne Hyperämie einzelner Gefässe, Papille nicht hyperämisch. Urin ohne Eiweiss und Zucker. Puls regelmässig, kräftig, 48. Partielle amnestische A phasie: Gabel: „Das ist zum Essen", Ring: „Das ist so an den Finger"; Löftel und Hut erkennt nnd benennt er richtig. Flustersprache rechts normal, links nicht gehört; Trommelfell rechts etwas eingezogen, unterbrochener Lichtretlex; links aus dem Ohr reichliche, stinke nde Eiterung, die ganze Tiefe ist von schmierigen Granulationen erfüllt, Warzenfortsatz links druckempfindlich; $W \mathrm{~L}+; \mathrm{Ri} K+, \mathrm{L}-$. Diagnose: Chron. Mittelohreiterung und schlafenlappenabscess links. Ich schritt sofort am

17. September zur Operation. Dauer 11/2 Stunde. Zuerst Radicaloperation des primären Ohrenleidens. Antrum mit Eiter und Granulationen erfült, sämmtliche Antrum-Wände cariös, Tegmen antri und tympani fehit ganz, Dura dort freiliegend, verdickt, mit festen Granulationen besetzt. Cholesteatom im Recess. epitymp.; Hammer und Amboss fehlen. Uebliche Lappenbildung.

Punction mit einer $2 \mathrm{gr}$ fassenden Spritze durch die verdickte Dura in den Schläfenlappen; die ganze Spritze füllt sich sofort mit gelbem, stinkendem Eiter von Milchconsistenz. Im unmittelbaren Anschluss hieran:

Trepanation a uf den linken Schläfenlappen von aussen ber. Ausmeisselung eines Knochenrechtecks aus der Squama, $3 \mathrm{~cm}$ lang, $11 / 2 \mathrm{~cm}$ hoch, $1 \mathrm{~cm}$ über dem höchsten Punkte des Porus acusticus, und zwar symmetrisch halb nach vorn und halb nach hinten von diesem gelegen. Dura pulsirt nicht. Einscbnitt durch die Dura in den Schlăfenlappen, reichlich $3 \mathrm{ctn}$ tief, kein Eiter. Erweiterung des Schnittes mit der Kornzange, dabei tritt zwischen deren Schenkeln der stinkende Eiter erst continuirlich, dann dem Pulse folgend stossweise hervor, im ganzen mindestens ein Esslöffel voll. Die Abscesshöhle hatte, soweit mit Sonde und Kornzange feststellbar, einen grössten Durchmesser von $5 \mathrm{~cm}$. Tamponade der Hohle mit 3 Jodoformgazestreifen, je $40 \mathrm{~cm}$ lang und $1,5 \mathrm{~cm}$ breit.

18. September. Der Kranke ist klarer. Puls über 60. Verbandwechsel; die gestrigen 3 Streifen, mit stinkendem Eiter durchsetzt, werden durch 2 ebensolche Streifen ersetzt; nach Herausnahme der ersteren entleert sich hinter ihnen her noch fast 1 Theeloffel voll Eiter. Bis

29. September guter Verlanf. Der Gestank der eingeführten Streifen lassst bald nach, es entleert sich auch nach ihrer Herausnahme hinter ihnen her kein Eiter mehr. Puls um 80, Temperatur normal. Kein Schwindel, kein Kopfschmerz. - A p hasie theil we is e noch vorhanden: Schlüssel, Uhr, Bürste benennt er richtig; Scheere: "Das ist zum Schneiden". Andere Gegen* stände betrachtet er erst lange und stellt dabei laut alleriei Betrachtungen an, um endlich das richtige Wort doch noch zu finden; z. B. Regenschirm: "Det is so zum Jehn, für den Fall, dass es regnen thut, ich weiss schon, ick habe ooch so ein Dings jehabt zu Hause" u. s. w., schliesslich sagt er: 
Schirm; oder Gabel: "Det is so cene Sorte zum Essen“, dann eine Reiho von unzusammenhängenden Worten, endlich: "Gabel“. Darnach benennt er mehrere Gegenstănde richtig; bält man ihm dann aber, also kaum nach $1 / 2$ Minute, die Gabel wieder vor, so weiss er sie von Neuem nicht zu bezeichnen. Auffällig ist eine gewisse Aufgeregtheit und Agilítät, Geschwätzigkeit und heitere Stimmung.

Augenhintergrund normal. Keine Sensibilitătsstörung. Einmal Nasenbluten.

Bakteriologische Unters uchung des am 17. entleerten Abscesseiters: im frischen Präparat ganz spärliche Kokken und ein feines Stäbchen mit Eigenbewegung, das zunächst den Eindruck von Proteus vulgaris macht; auf den Agar- und Gelatineculturen ist aber eine Art von Bacillus coli und eine grosse Kokkenart gewachsen.

30. September. Seit gestern auch rechts so schwerhörig, dass man sich nur schreiend mit ihm verständigen kann. 2 mal Erbrchen. Puls 58 , regelmässig.

2. October. Somnolenz. 53 Pulse.

3. October. $38 \mathrm{Pu} / \mathrm{se}$. Von der Hirnwunde aus wird nach hinten hin $6 \mathrm{~cm}$ weit mit dem Messer eingegangen, 3 Esslöffel Eiter entleert.

Darnach blieb der Kranke schläfrig, benommen. Puls wird immer langsamer.

6. October fruh $1 / 26$ Uhr Tod.

Obductionsbefund: Dura der oberen Pyramidenflache links in toto adhärent; strangartige Verwachsungen namentlich in der Umgebung des fehlenden Tegmen; Knochen nach rorn von der Tegmengegend noch rauh, cariös. Gehirnsubstanz darüber erweicht; über dieser erweichten Substanz eine grosse Abscessböhle, die fast die ganze Grosshirnhemisphäre einnimmt; von der Hirnsubstanz ist im Ganzen nur ein etwa $1 \mathrm{~cm}$ dicker Mantel erhalten, am Stirnlappen ist er noch etwa $3 \mathrm{~cm}$ dick. Keine Abscessmembran. Eine $\mathrm{Ab}-$ grenzung in der grossen Höhle, die darauf deutete, dass der am 17. September entleerte Eiter ans einer anderen Höhle als der am 3. October entleerte gestammt hätte, ist nicht zu entdecken.

Die Diagnose war in diesem Falle eine Art Schnelldi a gnose. Nachdem ich am 16 . September die stinkende Ohreneiterung links festgestellt hatte, fand ich am 17. September am Krankenbett den langsamen Puls und die Aphasie. Diese Symptome deuteten mit hoher Wahrscheinlichkeit auf einen linksseitigen Schläfenlappenabscess, und die iubrigen Erscheinungen passten alle ohne Weiteres in den Rahmen dieses Krankbeitsbildes, sodass hier die Diagnose schon vor dem operativen Eingreifen mit einer Sicherheit gestellt war, wie sie weitergehend bei einem Gehirnabscess kaum möglich ist. Auffallend ist das Fehlen jeder Lähmungserscheinung bis zum Tode trotz der aussergewöhnlichen Grösse des Abscesses, von Interesse ferner das Fehlen von Stauungserseheinungen am Augenhintergrunde trotz der ausgesprochenen sonstigen Erscheinungen erhöhten Hirndrucks (Pulsverlangsamung, Kopfschmerz), hervorhebenswerth auch die plötzlich auf dem bis dabin normal hörenden rechten Ohr auftretende hochgradige Schwerhörigkeit, die wohl auf eine Schädigung des Hörcentrums für das rechte Ohr im linken Schläfenlappen durch die vorwärtsschreitende Einschmelzung der linken Grosshirnhemisphäre zurückzufuhhren ist. 
Das rasche Weiterschreiten des eiterigen Zerfalls der Hirnsubstanz nach der ersten Operation am 17. September legt den Gedanken nahe, dass mit der Eröffnung des Abscesses und dem hierdurch herbeigefthrten freien Luftzutritt gewisse Mikroorganismen, die vorher unter unglinstigen Entwiekelungsbedingungen standen, jetzt plötzlich in günstigere Verhältnisse für ihre Vermehrung eintraten und nun von dem membranlosen Abscess aus die eiterige Einschmelzung der benachbarten Partien besohleunigten. Da ein solcher weiterer Zerfall glücklicherweise nicht jedesmal nach Eröffnung eines Hirnabscesses eintritt, so wäre abgesehen von den Fallen, in denen eine feste Abscessmembran einen gewissen Sclutz gegen weiteren Zerfall bildet, - anza. nehmen, dass solche Mikroorganismen eben nicht in jedem Falle vorhanden sind, wie wir denn bei anderen Hirnabscessen in der That nur eine Bakterienart, z. B. den Proteus vulgaris, ans dem Eiter ziuchten konnten, während hier Stäbchen und Kokken neben einander gefunden wurden.

Es w turde hiermit die Lehre von den aëroben und a na ëroben Mikroorganismen eine weitere praktische Bedeutung gewinnen.

\section{Doppelter Kleinhirnabscess rechts; einer davon operativ ent- leert; Tod am 20. Tage nach der Operation.}

Marie M., 18 Jahre alt, Putzmacherin. Seit frühester Kindheit aus unbekannter Ursache Ohrenlaufen rechts bis jetzt. Am 6. August 1899 Mittags erkrankte sie plötzlich an heftigen Kopfschmerzen mit Hitzegefühl und wurde deshalb in die Charité aufgenommen. Da gleichzeitig Blutung aus den Genitalien aufgetreten war, wurde sie, obwohl erst 8 Tage vorher die Menses dagewesen sein sollten, wegen Abortverdachtes auf die gynäkologische Abtheilung gebracht; zur Ohrenstation verlegt wurde sie am

9. August 1899. Grosses kräftiges Mädchen; innere Organe gesund. Sie ist apathisch, macht einen schwerkranken Eindruck, ihre Antworten erfolgen langsam. Sie klayt nur über heftige Kopfs chmerzen; der Kopf ist gegen Beklopfen stark, aber überall gleichmässig empfindlich. Keine Nackensteifigkeit, doch hält sic den Kopf stets mit auffallender Vorsicht unbewegt, da ihr jede Bewegung des Kopfes schmerzhaft ist. Sie kann allein gehen; hierbei fallt dio ruhige, eigenthümliche Haltung des Kopfes besonders auf. Beim Romberg'schen Versuch starkes Schwanken mit Neigung, nach hintenüber zu fallen. - Seit 2 Tagen ist sie heiser, ob infolge einer Stimmbandlähmung, ist leider nicht festgestellt. Sonstige Lăhmungserscheinungen fehlen, ebenso Sensibilitäts- und ataktische Störungen.

Augen: Rechte Pupille weiter als die linke, beide reagiren auf Licht. Beim Blick seitwărts and nach oben starker $\mathrm{Nystagmus.} \mathrm{Rochts} \mathrm{Papilla}$ n. optici mit undeutlichen Grenzen, trüb, Venen stark gefüllt; links Fundus normal. - Tremor manuum Patel larreftex nicht zu erzielen. Im Urin eine Spur Eiweiss, kein Zucker. Stuhl nicht angehalten. Zunge grau belegt, in Rachen und Nase nichts Auffalliges.

Ohren: Umgebung nirgends geschwollen. Rechts Warzenfortsatz und Bullus venae jugul. druckempfindlich. Flüstersprache links normal, rechts $15 \mathrm{~cm}$; WR + . Links Trommelfell leicht eingezogen, sonst alles normal. Rechts stinkende Eiterung, Totaldefect, nur kleiner oberer Rest mit 
Hammer, hinten oben weis sliche Massen. - Abends $39,4^{\circ}$, Puls $126-130$, regelmässig, klein.

10. August. Früh Temp. $39,8^{\circ} ; 10 \mathrm{Uhr} 37,4^{0}$. Puls 120 , regelmăssig. Grosse Hinfälligkeit und Schwäche. Auffallend starker Nystagmus. Diagnose: Chronische Mittelohreiterung rechts, vermuthlich mit Cholesteatom und Kleinhirnabscess. - Behufs Operation wurde, in Abwesenheit des Herrn Geh. Raths Trautmann, ich zugezogen (ich bin seit April 1899 nicht mehr Assistent der Klinik); ich schritt sofort

Frub $1 / 211$ Ubr zur Radicaloperation und Kleinhirnabscessoperation. Dauer zusammen $\% / 4$ Stunden. Als der Meissel im Proc. mast. etwa $1 \mathrm{~cm}$ weit vorgedrungen war, trat plötzlich eine Spur Eiter zu Tage; dasselbe wiederholte sich kurz darauf etwas weiter nach vorn, während nach hinten ein schwarzer Punkt, stecknadelkopfgross, sichtbar wurde; weiter nach hinten zeigte dann der Knochen missfarbige, grauschwarze Beschaffenheit. Antr., Adit. n. Recess. epitymp. mit einem wurstförmigen Cholesteatom erfüllt, so gross wie 3 neben ejnander gelegte Erbsen. Mediale und hintere Antrumwand cariös; Bogengang intact, sprang stark vor. Hammer gesund, langer Ambosschenkel cariös zerstört. Beim Vordringen nach hinten mit dem Löffel wurde eine nekrotische Fistelöfnung sichtbar, die in die hintere Schlăfengrube führte. Die Dura war anfangs stark zurückgesunken, so dass man durch die Fistel in eine dunkle Höhle hineinsah; allmählich aber kam die Dura vor und legte sich gegen die künstlich vergrösserte Fistelöffnung. Dura misstarbig, grüngelb, auch über dem Sinus. Punction mit einer 2 gr fássenden Spritze durch die Dura ins Rleinhirn, alsbald füllt sich die ganze Spritze mit stinkendem, dünnem, graugrünem Eiter.

Gegen den so festgestellten Kleinhirnabscess wird jetzt von aussen her in der bei uns ublichen, anderwärts (l. c., oben) geschilderten Weise vorgegangen. Kunochenöfinung im Hinterhaupt wird $3 \mathrm{~cm}$ lang, $1,5 \mathrm{~cm}$ hoch ange. legt. Auf Einscbnitt mit dem Messer entleert sich nichts, erst als mit der Kornzange tief nach vorn und unten eingegangen wird, entleeren sich zwischen ihren Branchen 2-3 Esslöffel voll stinkenden, dünnnen, graugrünen Eiters. Drainage des Kleinhirns mit Jodoformgazestreifen.

Die mikrosk op is ch-bakteriologis che Untersuchung des Kleinhirneiters ergab: Detritus, nur ganz vereinzelte Zellen; massenhafte Kokken, meist Diplokokken, einzelne mit Hof, dann auch Streptokokken und vereinzelte kurze Stäbchen; auf Agar wuchsen hauptsächlich Culturen von Streptokokken, die unter dem Mikroskop lange Ketten bildeten, ferner von Proteus und schliesslich auch von Diplokokken.

Nach der Operation und am Tage darauf fühlte sich die Pat. wohler, die Temperatur fiel zur Norm ab, doch schon am 12. August überschritt sie wieder $38^{\circ}$ und stieg am 13. früh wieder auf $40,6^{\circ}$; am 14 . unter $38^{\circ}$; am 15. früh 40,4 mit Schüttelfrost; am 19. wieder Schüttelfr ost, $40,6^{\circ}$; von da an täglich hohes Fieber mit Remissionen bezw. Intermissionen; Puls $z$ wischen 104 und 160, aber immer regelmässig. Das Befinden der Pat. war vom 12, an wieder schlecht, sie klagte über Kopfschmerzen, war apathisch, jammerte vor sich hin und bot dieselben Symptome, wie vor der Operation, insbesondere jene auffallende Schmerzhaftigkeit bei jeglicher Bewegung des Kopfes; nur die Stauungserscheinungen am rechten Augenhintergrunde waren verschwunden. Am 17. traten Esscheinungen einer Endocarditis, am 20, die einer Lungenmetastase, später wiederholt Erbrechen und schliesslich Durchfälle hinzu.

Bei den fast tăglich vorgenommenen $\mathrm{V}$ e rbandwechseln entleerte sich regelmässig nach Entfernung der behufs Drainage in das Kleinhirn eingeführten Jodoformgazestreifen entweder spontan hinter diesen her oder nach vorsichtigem Eingeben mit der Kornzange wieder Eiter, mehrfach bis zu einem Esslöffel voll. Am 13. August stiess sich beim Verbandwechsel die der Wundhöhle zugekehrte Wand des Sinus nekrotisch ab; der Sinus war nach oben and unten bin fest verschlossen, Eiter entleerte sich aus ihm nicht. Am 18. August entleerte sich beim Verbandwechsel Eiter aus der Gegend zwischen Dura und hinterer Pyramidenfläche. 
Die Pat. war vom 20. August an meist comatös; wiederholte Collapszustände wurden durch zahlreiche Campherinjectionen äberwunden. Am 30. August Nachmittag $1^{1 / 2}$ Uhr trat, nachdem am Morgen noch einmal in Narkose wit weiterer Freilegung und Ausräumung des Sinus sowie mit Eröfinung des Abscesses auch von der Radicaloperationswunde her vorgegangen worden war, der Tod ein.

Obductionsbefund: Arachnoidea an der Basis zart. Rechte Kleinhirnhälfte mit dem Sinus transversus in der Gegend des Sulcus sigmoid. verklebt, aber doch leicht abtrennbar. Sinus missfarbig (uber seinen Inbalt ist im Protokoll leider nichts gesagt); Knochen im Sulc. sigm. cariös, mit Granalationen besetzt. Das Kleinhirn ist von hinten und von vorn her durch Operation eröffnet; von beiden Stellen her gelangt man in ein und denselben Abscess (über Grösse und Membran ist nichts gesagt); neben dem operativ eröffneten befindet sich noch ein zweiter, nicht eröffneter, kirschengrosser Abscess mit eingedicktem Eiter. - Abscessus cerebelli dextri duplex, Thrombosis sinus transversi. Pneumonia metastatica dextra, Empyema pleurae dextrae.

Die Eiterung war hier vom Mittelohr quer durch den Warzenfortsatz auf einem bei der Operation zum Theil noch erkennbaren We ge zunächst gegen den Sinus hin vorwärts geschritten und hatte dann weiterhin über den thrombosirten Sinus hinaus das Kleinhirn erreicht. Auffallend ist, dass der Zerfall der Thrombusmassen im Sinus und damit die pyämischen Erscheinungen erst nach der operativen Entleerung des Hirnabseesses einsetzten.

Hervorhebenswerth erscheint mir weiter die grosse Sch mer zhaftigkeit des Kopfes bei jeder Bewegung und die hierauf zurtickzuftihrende vorsichtige Vermeidung jeder Bewegung des Kopfes, auch in Bettruhe, sowie das Verhalten des $\mathrm{Pulses}$, der nicht verlangsamt und unregelmässig, sondern stark beschleunigt und stets regelmässig gefunden wurde - Punkte, auf die ich unten noch zurückkommen will. - Schliesslich sei noch ausdrücklich darauf bingewiesen, dass neben dem operativ eröffneten bier noch ein $z$ weiter, nicht eröffneter Kleinhirnabseess in derselben Hemisphäre vorhanden war; in dieser Du plieität dürfte das unveränderte Fortbestehen fast aller Symptome nach und trotz der Entleerung des einen Abscesses, wenigstens zum Theil, seine Erklärung finden.

Die ungentugende Absaugung des Eiters aus dem Abscess, die sich bei jedem Verbandwechsel in spontanem oder mit der Kornzange leicht herbeizuftuhrendem Nachsturzen von Eiter hinter den eingeführten Gazestreifen her kund gab, legt die Frage nahe, ob bei solchen tief sitzenden Abscessen nicht doch besser mit Röhren zu drainiren wäre, wiewohl die Nachtheile dieses Verfahrens (Druck der Röhre auf die Abscesswand und infolgedessen Decubitus an der Druckstelle) uns bis jetzt immer davon abgehalten haben. Auch könnte man daran denken, 
dass unsere Knochenöffnung - 1,5:3,0 $\mathrm{cm}$ - doch vielleicht zu einer ansreichenden Entleerung zu hoch gelegen und zu klein gewesen wäre; indess erscheint es mir fraglich, ob eine bessere und vollständigere Entleerung des Eiters nach hinten hin würde möglich gewesen sein, auch wenn man die Knochenöffnung weiter unten und doppelt so gross angelegt hätte: eher scheint mir in Fällen wie dem vorliegenden, wenn selbst beim 4. und 5. Verbandwechsel noch der nachstïzende Eiter das Ungenügende der Drainage nach hinten hin darthut, der Versuch angezeigt, dem Eiter nach der Radicaloperationswundhöhle hin durch weitere Abmeisselung der hinteren Pyramidenwand freieren Abfluss zu verschaffen, namentlich wenn, wie bier, das Fehlen von Hirnprolaps an der äusseren Hirnwunde zu der Hoffnung berechtigt, dass ein solcher auch in die Radicaloperationswundhöhle hinein nicht stattfinden werde.

\section{Doppelter Kleinhirnabscess links; operativ in 2 Eingriffen entleert; Heilung.}

Frieda Sch., 10 Jahre alt, Maurerstochter. - 1897 Fall auf den Kopf; seitdem öfters Koptschmerzen. Mitte October 1898 ohne bekannte Ursache Ohrenlaufea liaks. Am 1. November 1898 Erbrechen, Kopfschmerz; am 2. November Nasenbluten. Seit 9 . November heftige Kopfschmerzen.

12. November 1898. Aufnahme in die Charité. Grosses, leidlich kräftiges Kind. Klagt nur über Kopfschmerzen. - Temp. 37,9 ${ }^{\circ}$, Puls 72 , nicht ganz regelmässig. Schwerkrank, benommen. Beim Gehen $\mathrm{Sch}$ wanken nach der linken (kranken) Seite; beim Romberg'schen Versuch deutliches Schwanken. Bewegungen des Kopfes, besonders Beugen nach vorn, schmerzhaft; der $\mathbf{K}$ opf wird deshalb beim $G$ ehen steif und unbewegt gehalten; dieses Verhalten erweckt Verdacht auf Nackensteifigkeit, doch ist diese zweifellos nicht vorhanden. - Reichliche Eiterung aus dem linken Ohr.

Augenhintergrund: Beiderseits Schlangelung der Venen, links trübe, verwaschene Papillengrenze. Nystagmus. . Linke Pupille weiter als die rechte.

14. November 1898. Lu mbalp un ction: Wasserklare Flüssigkeit, aus der sich keine Mikroorganismen züchten lassen.

16. November 1898. Verlegung zur Ohrenstation. Temp. $35^{\circ}$, Puls wechselnd, 72-104, zeitweise etwas unregelmässig. - Nach jeder Nahrungsaufnahme Erbrechen, daher Eroührung durch Clysmata. Starker Foetor ex ore, Zunge dick gran belegt, Lippen trocken, rissig. Schwerkranker, zeitweilig soporöser Zustand, aus dem das Kind aber durch lautes Anreden za erwecken ist; sie giebt dann klare Antworten. Keine Aphasie. Delirirt und phantasirt viel vor sich hin, ist zeitweise äusserst redselig und wirft Zusammenhängendes und Ungereimtes durcheinander; dazwischen hindurch schreit sie manchmal laut auf und klagt über Schmerzen im Hinterkopf, in der Nackengegend und im Rücken, sowie über Schwindel; letzterer ist nicht näher zu prüfen, beim Versuch, sie aufzurichten, schreit sie laut. Keine Lähmungserscheinungen; Facialis in Ordnung. Ob ataktische Störungen vorliegen, lässt sich nicht prüfen. Keine Nackensteifigkeit. Halsgefässe nicht strangartig durchzufühlen, Gegend des Foramen jugulare nicht druckempfindlich. - Hörprüfung nicht möglich.

Ohren: Links beim Abtupfen reichlicher Eiter, nicht stinkend, äusserer Gebörgang in der Tiefe von hinten her verengt, Trommelfell geröthet, verdickt, vorn unten kleiner Defect. Rechts ist das Trommelfell stark eingezogen, ohne Lichtkegel, sonst normal. - Ich schritt sofort am 
16. November 1898 früh $10 \mathrm{Uhr}$ zur Antrumaufmeisselung links und zur Kleinhiraboscessöf fung links

in einer Sitzung. Dauer $7 / 4$ Stunden, Narkose ruhig, $22 \mathrm{~g}$ Chloroform.

Antrumwände morsch, Antrum und die ubrigen Warzenfortsatzzellen mit zähem Schleim und verdickter, granulirter Schleimhaut ausgefüllt. Es wird absichtiich nach oben bis zur Dura, nach hinten bis zum Sinus vorgegangen, Dura und Sinus je 1 gcro weit freigelegt. Punction durch die Dura in den Schläfenlappen făllt negativ aus. Darnach Punction am Siuus vorbei ins Kleinhirn; Die Spritze aspirirt dicken, grünlichgelben, nicht stinkenden Eiter.

Eröfnung des so festgestellten Kleinhirnabscesses von hinten her in der bei uns üblichen Weise (s. die Eingangs citirte Arbeit utber einen Kleinhirnabscess). Beim Einschneiden mit dem Messer ins Kleinhirn entleert sich vichts, erst beim Erweitern der Hirnwunde mit der Kornzange nach innen und vorn tritt stossweise mit dem Puls grüner Liter, mindestens $1^{3 / 2}$ Fss10ffel, zu Tage, anfangs dick, zuletzt fast serös dünn. Tamponade und Drainage der Abscesshohle mit Jodoformgazestreifen, sie fasst 3 Streifen von $2 \mathrm{~cm}$ Breite und je $9 \mathrm{~cm}$ Länge. - Der Abscesseiter enthielt neben Eiterkörpercben eine Reincultur von Streptokokken.

Nachmittag $38,8^{\circ}$. Puls 120, regelmässig. Klares Bewusstsein; noch sehr redselig.

Vom 17. November an während des ganzen weiterea Verlaufs fieberfrei, aber nicht subnormale Temperatur.

Die Besserung nach der Operation war keine vollkommene. Puls zwischen 102 und 120. Fortgesetzt Bchwindel beim Versuch, sich aufzurichten. Nach versuchter Nahrungsautnahme immer noch Erbrechen, daher Ernãhrung lediglich durch Klystiere bis 30 . November; bis 27 . November ausserdem auch spontan ofter Erbrechen, bis zu $6 \mathrm{mal}$ an einem Tage. Stubl erfolgt nur auf Eingiessung, später auf Inf. Rhel, erst vom 13. Januar 1899 an spontan. Liegt meist mit gegen den Unterleib angezogenen Knieen im Bett. - Am 18., 21., 23. und 25. November Verbandwechsel. Die drainirenden Gazestreifen sind immer vollig durchtränkt, doch entleert sich hinter ihnen her kein Eiter mehr. Am 25. wird die prolabirte Kleinhirnmasse, etwa 3 Erbsen gross, abgetragen, Kleinhirusubstanz odematös durchtränkt.

27. November 1898. Sopor, Puls 66-80, nicht ganz regelmässig. Urin unter sich gelassen. - A ugenhintergrund: Links stark geschlängelte Venen, rechts keine Staunugserscheinnngen; starrer Blick. - Verband wechsel ohne Narkose, dabei vôllig apathiseh, onne Schmerzen. Punction erst naeh vorn oben, negativ; dann Punction nach hinten oben: die Spritze aspirirt seröse, trübe, gelbliche Flüssigkeit. Erweiterung nach dieser Richtung mit der Kornzange; stossweise tritt erst dunne Flüsigkeit wie bei der Punction, dahinterher dicker, gelber, nicht fötider Eiter, schliessjich wieder etwas seröse Flüssigkeit zu Tage, im ganzen wenigstens 2 EssIoffel. Die Abscesshohle ist jetzt viel grösser als nach der ersten Fröffung; sie fasst 3 Jodoformgazestreifen von $2 \mathrm{~cm}$ Breite und je $15 \mathrm{~cm}$ Länge.

Im Eiter wie in der serösen Flüssigkeit zeigen sich im Ausstrichpräparat wie in der Cultur von Mikroorganismen nur Streptokokken.

Fine Stunde nach der Abscessentleerung ist das Mädchen bei klarem Bewusstsein; freier, nicht mehr starrer Blick. - Die einzelnen Krankheitserscheinungen schwinden allmahlich in den folgenden Tagen, Kopfschmerzen und Schwindel lassen nach, das Erbrechen hört auf; am 30. November erste Nahrungsaufnahme per os, bis 5. December noch theilweise Ernährung durch Klystiere, von da an nur uoch per 0 s. Am längsten bielt die Stubiverstopfung an (bis 13. Januar 1599). Auffallend war bei allen folgenden Verbandwechseln eine stets beim Einführen der neuen Streifen in die Hirnwunde auftretende Schmerzenptindung in der Stirn uber dem linken Auge.

6. December 1898 . Im Wundsecret keine Streptokokken mehr nachzuweisen. - Das Kind ist bei guter Stimmung, lacht und unterhält sich. Rege Esslust. - Kein Nystagmus mehr, Augenhintergrand frei. - Puls noch um 100 , regelmässig. 

60 Pfund.

Vom 2. Januar bis 28. März 1999. Gewichtszunahme von 52 auf

23. Februar 1899. Kleinhirnwande verheilt. Heilungsdauer $31 / 4$ Monate.

30. März 1899. Antrumwunde geheilt.

4. April 1899. Flustersprache links $5 \mathrm{~m}$, rechts normal. Das Kind ist ohne alle Beschwerden. Geheilt entlassen.

Mitte Juli 1899 brach die Antrumwunde wieder auf, musste noch einmal ausgekratzt werden und war erst Ende 1899 wieder vollig verheilt; die Kleinhirnwunde blieb aber seit 23. Februar fest verheilt. - Anfang Mai 1900 stellte sich das Mädchen mir wieder vor. Sie ist obne Beschwerden, hat sich kräftig entwickelt und macht in der Schule gute Fortschritte; trotz der langen Schulversäumniss ist sie zu Ostern versetzt worden uud ist sogar in der Klasse eine der ersten. - Kein Sausen, kein Schwindel, kein Kopfschmerz; nie Krampfanfälle. Romberg völlig negativ. Trommelfell rechts ganz normal, links leicht getrübt, sonst ebenfalls normal. Hörvermögen fur Flüstersprache beiderseits absolut normal; Stimmgabel tönt vom Scheitel noch nach links. Druck auf die Hirnoperationsnarbe nicht schmerzhaft, löst keinen Schwindel und auch nicht jenen Stirnschmerz mehr aus.

Der Fall ist insofern bemerkenswerth, als hier durch die Operation eine völlige, ideale Heilung erzielt worden ist. Es ist nicht die geringste Spur einer krankhaften Störung zurückgeblieben, weder von Seiten der Ohren, noch anch von Seiten des Gebirns, trotz jener Abtragung einer nicht unbeträchtlichen Quantität der Kleinhirnmasse am 25. November 1898. Da ich bei der Operation der Meinung war, eine acute Mittelohreiterung vor mir zu haben, begnügte ich mich mit der AntrumAufmeisselung - also ohne Radicaloperation -, und die blosse Antrum-Eröffnung hat zur gänzlichen Beseitigung der Ohreneiterung und zur völligen Wiederherstellung des normalen Hörvermögens geflihrt. Bei der letzten Vorstellung des Kindes Anfang Mai 1900 gab mir aber die Mutter nachträglicb an, dass im Alter von 7 Jahren das Mädchen Scharlach durchgemacht und darnach "nur ein paar Tage" Ausfluss aus dem linken Ohre gehabt hat. Hiernach kann von einem rein acuten Charakter der Obreneiterung nicht mehr die Rede sein, die Eiterung muss als zum mindesten auf chronischer Basis beruhend angesehen werden, und hiermit ist anzunehmen, dass auch die Entwickelung der Kleinhirnabscesse hier lange vor dem Auftreten der ersten manifesten Erscheinungen zu Beginn des Novembers 1898 ibren Anfang genommen hat. Bemerkenswerth ist weiter, dass wir es bier zweifelsohne mit einem doppelten Abseess zu thun gehabt haben. Die Richtung der den Abscess eröffnenden Kornzange war bei der ersten Operation nach vorn und innen, bei der zweiten am 27. November naeh hinten und oben. Die erste Abscesseröffnung brachte nicht die zu erwartende Besserung; erst durch die zweite Entleerung wurde, nach Her- 
stellung einer gemeinsamen Abscesshöhle, die eigentliche Heilung eingeleitet.

Ueber den Weg, den die Eiterung vom Mittelohr zum Kleinhirn genommen hat, brachte die Aufmeisselung des Warzenfortsatzes direct keinen Aufschluss. Eine Betheiligung des Sinus und der Weg tiber diesen hinweg darf nach den klinischen Erscheinungen und nach dem Befunde bei der Operation mit Bestimmtheit ausgesehlossen werden. Dass die Eiterung sich einen directen Fistelgang durch den Knochen neu gebildet und so das Kleinhirn erreicht hätte, darf bei dem Mangel grösserer cariöser Zerstörungen im Warzenfortsatz ebenfalls als ausgeschlossen gelten. Es bleibt also nur der Schluss tibrig, dass der eiterige Process entweder duroh den Meatus acustic. internus oder durch den Aquaeductus vestibuli vorwärts geschritten sei; gegen den ersteren Weg spricht die Thatsache, dass im linken Facialisgebiet nie eine Störung wahrzunehmen gewesen und eine völlige Wiederherstellung des Hörvermögens auf dem linken Ohre eingetreten ist, und so hat am meisten die Annahme fur sich, dass es der Aquaeductus vestibuli war, durch den sich das infectiöse Material zum Kleinhirn hin Bahn gebrochen hat.

Auffallend war das vielfach aufgeregte Wesen, das Phantasiren und Deliriren und die zeitweise auftretende Redseligkeit des Kindes vor der Operation, während man sonst bei Kleinhirnabscessen doch melr ein schläfriges, apathisches Wesen der Kranken zu sehen gewöhnt ist. Diese Aufgeregtheit muss wohl als ein Symptom der beginnenden Erhöhung des allgemeinen Hirndrucks angesehen werden, obgleich Pulsverlangsamung dabei fehlte. Jedenfalls ist dieses Erregungsstadium kein constanter Vorläufer der eigentlichen Hirndrucksymptome bei Hirnabscessen; es erinnerte daher in unserem Falle eher an Meningitis. Zudem liessen die Schmerzen bei Bewegungen des Kopfes an beginnende Genickstarre denken, und anch das mehrfach beobachtete laute Aufschreien legte den Gedanken an eine Meningitis nahe. Bei genauerer Untersuchung zeigte sich aber, dass Nackensteifigkeit nicht vorlag, dass vielmehr nur die Zunahme der Kopfschmerzen bei Bewegungen des Kopfes das Kind zu möglichst ruhiger Haltung des Kopfes und zu Widerstand beim Versuch, ihn passiv zu bewegen, veranlassten; ausserdem fehlte höheres Fieber, auch fiel die Lumbalpunction negativ aus, und der Schwindel und der Nystagmus wiesen daher - in Verbindung mit allen ubrigen Symptomen, dem schwerkranken $\mathrm{Zu}$ - 
stande u.s.w. - viel mehr auf einen Kleinhirnabscess hin. Gestittzt wurde die Annahme eines solchen nach meinen Erfahrungen auch noch durch die linkerseits stärkeren Stauungserscheinungen am Augenhintergrunde, die ich, wie ich später noch näher erläutern will, als eine Folge localen Druckes auf die reichlichen venösen Blutbahnen in der hintern Schädelgrube, und zwar hier der linken, ansprechen zu müssen glaube.

Bei alledem stand, wie nicht zu leugnen, die Diagnose hier auf recht schwachen Füssen und blieb zweifelhaft, bis die Punction in das Kleinhirn naoh beendeter Antrum-Aufmeisselung die erhoffte Sicherheit brachte. Bis dahin wiesen, wie so oft, die Symptome eigentlich nur anf eine schwere cerebrale Complication hin, wobei Sinusthrombose mit Bestimmtheit und Meningitis allenfalls noch mit einiger Wahrscheinlichkeit ausgeschlossen werden konnte; mehr liess sich zunächst nicht sagen. Unser Vorgehen - Mastoidoperation, Freilegung der mittleren und hinteren Sehädelgrube und Probepunction in Schläfenlappen und Kleinhirn - dürte fur jeden derartigen Fall als das rationellste zu empfehlen sein.

Ich habe im Vorstehenden, was die Falle etwa im Einzelnen Interessantes boten, am Sehlusse jeder Einzelschilderung hervorgehoben; es bleibt mir ubrig, noch einige allgemeine $\mathrm{Be}$ merkungen anzuschliessen, zu denen mir meine fruher beschriebenen bezw. nur beobachteten und die heute geschilderten Fälle, in Verbindung mit dem Studium der Litteratur der otitischen Hirnabscesse, Veranlassung geben.

Die grosse Mannigfaltigkeit der Symptome, die bei Gehirnabscessen vorkommen können, sowie die Versehiedenheit in der Zusammenstellnng und Gruppirung der einzelnen Symptome in den einzelnen Fällen und die dadurch bedingte erhebliche Verschiedenartigkeit des Krankheitsbildes bei Gebirnabscessen drängen jedem, der sich mit ilınen beschäftigt, die Frage auf: Worin liegt der Grund zu dieser Mannigfaltigkeit und Verschiedenartigkeit?

Nach einer Richtung bin ist die Antwort auf diese Frage leicht gegeben. Die einzelnen Bezirke des Gehirns haben die verschiedensten Functionen, und da ist es selbstverständlich, dass je nach dem Sitze der Erkrankung in dem einen oder anderen Bezirke und je nach der Ausdehnung der Erkrankung ther einen oder mehrere Bezirke die Erkrankung hier diese, dort jene Störungen als Krankheitssymptome zur Folge haben wird. Manche 
Verschiedenheiten der Symptome sind aber mit dem regionären Sitz und der Grösse des Abscesses nicht erklärt.

Wie kommt es nämlich, dass oft ein verhältnissmässig kleiner Hirnabscess schwere Symptome erhöhten Hirndrucks verursacht, während ein viel grösserer Abscess ganz und gar ohne Drucksymptome verläuft? Selbstrerständlich können hier ausserhalb des Abscesses liegende Factoren mitsprechen, z. B. vermehrter Liquor cerebrospinalis infolge seröser Meningitis; ebensogut aber können solche ausserhalb liegende Factoren fehlen. Und dann reicht es zur Beantwortung unserer Frage nicht ans, nur den regionären Sitz des Abscesses in Betracht zu ziehen, wir müssen vielmehr den Sitz des $A$ bsoesses auch rom histologischen Standpunkte aus untersuchen: sitzt er in dem eigentliehen Hirngewebe oder sitzt er in dem an sich ja sehr spärlichen, durch entzündliche Vorgänge aber schnell und stark vermehrbaren bindegewebigen Stitzzgerüst, in dem interstitiellen Gewebe des Gehirns? und gleichzeitig ist weiter zu fragen: handelt es sich um eine eiterige Einschmelzung von Gehirnsubstanz, also um einen destructiven Vorgang, oder liegt eine Ansammlung neugebildeten Eiters in dem bindegewebigen Stützgerüst des Gehirns, also ein productiver Process, vor?

Diese Fragen führen mich dazu, rom histologischen Standpunkte aus grundsätzlich zwei Arten von Gehirnabscessen zu unterseheiden; die eine möchte ich den parenchymatösen, die andere den interstitiellen Hirnabscess nennen, ersterer ist der eigentliche Abscessus cerebri bezw. cercbelli, letzterer im Grunde genommen nur ein Abscessus in cerebro bezw. in cerebello.

Der parenchymatöse Gehirnabseess ist aufzufassen als das Resultat einer eiterigen Einschmelzung oder brandigen Zerstörung der eigentlichen Hirnsubstanz, also eines degenerativen oder destructiven Vorganges; die von dem eiternden Ohre aus eindringenden Infectionsstoffe bewirken nicht eine entzlindliche Answanderung von Leukoeyten, sondern sie fuhren zum Zerfall, zur Zerstörung der von ihnen heimgesuchten Hirnpartien. Dementsprechend ist auch der Inhalt eines solchen Abscesses nicht eigentlich Eiter, sundern Brandjauche, I chor.

Anders der interstitielle Gehirnabscess. Er ist das Product eines durch die von der Ohreiterung her eindringenden schädlichen Keime hervorgerufenen Entzündungsvorgangs im interstitiellen Bindegewebe, eines productiven oder auch formativen 
bezw. exsudativen Processes; entzindliche Neubildung und Vermehrung dieses Bindegewebes mit Auswanderung von Eiterkörperchen in den entzlindeten Bezirk hinein und damit Neubildung eines Herdes von rein em Eiter ist hier das Charakteristisehe. Während bei dem parenchymatösen Abscess also etwas bereits Vorhandenes der Zerstörung bezw. einer Umwandlung anheimfällt, wird bei dem interstitiellen Abscess etwas vorher noch nicht Vorhandenes neu gebildet und dem bis dahin vorhandenen Schädelinhalte neu hinzugefugt.

Warum die von der benachbarten Ohreiterung eindringenden Schädlichkeiten das eine Mal einen entzlindlichen eiterigen Vorgang im bindegewebigen Stützgerüst der Hirnsubstanz und das andere Mal eine eiterige Einschmelzung bezw. brandige Zerstörung der Hirnmasse selbst herbeiführen, vermag ich nur vermuthungsweise $z u$ beantworten. Jedenfalls spielt dabei die Art der eindringenden Schädlichkeit eine massgebende Rolle. Handelt es sich um die als Eitererreger bekannten Kokkenarten, so wird eine interstitielle Eiterung die Folge ihrer Einwanderung sein; dringen aber Fäulnissbakterien vom Ohre aus in das Schädelinnere, so wird dies fauligen Zerfall und Brand der unter ihren Einfluss gerathenen Partien zur Folge haben; möglich auch, dass es sich im letzteren Falle nicht oder nicht immer oder nicht allein um eine schädliche Lebensäusserung der dabei anzutreffenden Mikroorganismen, sondern vielleicht auch um eine Art chemischer Wirkung schädlicher nicht lebender Stoffe handelt.

Mit der Unterscheidung dieser zwei Kategorien von Hirnabscessen nach ihrem histologischen Sitz ist dann auch ganz von selbst eine Reihe weiterer Differenzpunkte zwischen ihnen gegeben.

Die erste Verschiedenheit habe ich schon angedeutet, sie betrifft ihren Inhalt. Der parenchymatöse Abseess enthält dinne, grüliche, stinkende Jauche, Ichor, der interstitielle Abscess dicken, rahmigen, nicht fötiden Eiter, Pus. Im Ichor finden wir mikroskopisch Detritusmassen, nur wenig Eiterkörperchen, nur wenig Kokken, dafür aber zahlreiche Fäulnissbakterien, insbesondere den Proteus vulgaris, am Ichor ist ferner charakteristiseh sein Gestank. Dagegen enthält der Eiter des interstitiellen Abscesses Kokken, z. B., wie in unserem Falle IV, eine Reineultur von Streptokokken, und daneben zahllose Eiterkörperchen; er ist geruchlos oder von fadem Eitergeruch. 
Der interstitielle Abscess hat eine bindegewebige Membran, eine Balgkapsel von bindegewebiger Struetur, herrührend von den massenhaft entzündlich neugebildeten und vermehrten Bindegewebselementen des an und für sich tuberaus feinen und spärlichen interstitiellen Neuroglia- Hirnstützgertists, in welehe sich der Strom der eiterbildenden Lenkocyten ergossen hat, -der parenchymatöse Abscess ist obne solche bindegew ebige Membran und wird höchstens durch eine infiltrirte, derbe Randpartie, die ja dann auch den Eindruck einer Balgkapsel machen kann, ohne aber bindegewebigen Charakter zn haben, von dem umgebenden gesunden Hirngewebe abgegrenzt. Hiermit findet die Thatsache ihre Erklärung, dass die eine Balgkapsel von ihrer Umgebung leicht abzuziehen ist - das ist die echte, bindegewebige Membran -, während die andere sich nur in kleinen Fetzen unter Zerreissungen von ihrer Umgebung lostrennen lässt - das ist die Psendobalgkapsel des parenchymatösen Abscesses, die man allenfalls als eine "ichorrhogene" Membran bezeichnen könnte -; viele parenchymatöse Abscesse sind aber überhaupt ohne jede Kapsel. Ferner liegt hierin die ungezwungene Erklärung für die Thatsache, dass ein abgekapselter Abscess nicht etwa bei einem Stillstand seines Wachsthums angekommen zu sein braucht, sondern ruhig weiter wachsen kann, und schliesslich auch dafür, dass eine vorhandene Balgkapsel durchaus nicht allemal auf ein hohes Alter und umgekehrt das Fehlen einer Membran ebensowenig immer auf die frische Entstehung des Abscesses schliessen lässt.

Wichtiger als diese pathologiseh-anatomisehen und bakteriologischen Untersehiede sind aber die Differenzen, die wir auf klinischem Gebiete zwisehen den beiden Arten von Hirnabscessen feststellen können.

Der parenchymatöse Abscess verläuft, da es sich ja um einen eigentlich entzündlichen Vorgang hier nicht handelt, obne Fieber, ja es können subnormale Temperaturen vorkommen, wie sie bei Hirnabscessen in der That nicht selten beobachtet werden, oder aber es tritt, wenn die jauchigen Stoffe in die Lymph- und Blutbahnen gelangen, Fieber von ausgesprochen ichorrhä misch em (septichämischem) Charakter auf, wie ebenfalls bisweilen $\mathrm{zu}$ beobachten ist.

Der interstitielle Abscess dagegen, als das Resultat eines entzlindlichen Vorganges, wird immer von etwas Fieber begleitet sein, das aber, entsprechend der verhältnissmässig ge- 
ringen Ausdehnung des entzindlichen Herdes der Gesammtmasse des Körpers gegentiber, nur mässig sein und sich im Allgemeinen, wenn nicht andere temperatursteigernde Factoren hinzukommen, in abendlichen Erhöhungen der Körperwärme auf 37,2-37,8 oder $38,0^{0}$ äussern wird, wie dies thatsächlich ja bei Gehirnabscessen so oft festzustellen ist.

Der wichtigste Untersehied zwischen beiden Abscessarten liegt aber auf dem Gebiete der Erscheinungen des erhöhten Hirndrueks.

Es ist klar, dass die Neubildung eines Eiterherdes im interstitiellen Bindegewebe des Gehirns dureh frisehe Zufuhr von Eiterzellen, die unter allmäliger Auseinanderdrängung der benachbarten Hirnpartien, aber - zunächst wenigstens - unter völliger Erhaltung dieser Partien ihrer Masse und ihrem Vo. lumen nach vor sich geht, raumbeschränkend wirken und eine allgemeine Erhöhung des intracraniellen Druckes zur Folge haben muss, wenn auch diese Druekerhöhung fur's erste noch keine nach aussen hin wahrnehmbaren Symptome zu Stande zu bringen braucht, da sie zunächst durch das Ausweichen des Liquor cerebrospinalis nach dem etwas erweiterunsfähigen Duralsacke des Rückenmarks hin einen gewissen Ausgleich erfährt. Ebenso klar aber ist es, dass ein Jaucheherd, der sich durch Einschmelzung einer stets seiner eigenen Grösse entsprechenden Menge von Hirnsubstanz bildet, bei dem also die Menge des neu auftretenden Abseessinhaltes stets gleichen Sehritt hält mit der Menge der daftur in Wegfall kommenden Hirnmasse, eine Erhöhung des allgemeinen intracraniellen Druekes nicht zur Folge haben wird. Beim interstitiellen Abscess haben wir also allgerneine Hirndrucksymptome, beim parenchymatösen nicht. Verlangsamter Puls, allgemeiner Kopfschmerz, allgemeine Percussionsempfindlichkeit des Schädels, das Gefühl, als wollte es den Schädel auseinandersprengen, Staungserscheinungen am Augenhintergrunde beiderseits, Mydriasis beiderseits, Uebelkeit und Erbrechen, Irrereden in den früheren, Schlafsucht und Bewusstlosigkeit in den späteren Stadien, alles das sind Symptome, die wir bei dem interstitiellen Abscess erwarten müssen, während wir sie beim parenchymatösen Abscess vermissen werden.

Nun können aber auch bei letzterem Hirndruckerscheinungen vorkommen; doch handelt es sich dann nicht um die Folgen allgemein erhöhten, sondern localen Hirndrucks. Es leuchtet 
ein, dass ein durch Einschmelzung der Hirnsubstanz entstandener Eiterherd sich hinsichtlioh des Druckes, den er auf seine Umgebung ausübt, wie ein fremder Körper, also ganz anders verhalten wird, als die Hirnsubstanz, die vorher an seiner Stelle war; diese war dureh Gefässe und Faserungen anf das innigste mit ihrer Umgebung verbunden und von gleicher Consistenz sowie von gleichem specifischem Gewicht wie die umgebenden Hirnpartien; das neugebildete Einschmelzungsproduct dagegen ist aus aller organischen Verbindung mit der Umgebung gelöst, ist von dunnerer Consistenz, also flussiger als die Hirnsubstanz und von anderem specifischem Gewicht als die Hirnmasse. Sonach ist es begreiflich, dass der Eiterherd auf seine Umgebung und zwar zumeist wohl auf die unter ihm liegenden Abschnitte eine Art todten Druckes ausüben wird. Dann werden locale Druoksymptome die Folge sein: 'einseitige Staungserscheinungen am Auge - diese namentlich beim Sitz des Abscesses im Kleinhirn, wo die zahlreichen, im Gebiete der hinteren Schädelgrube anzutreffenden venösen bezw. sinuösen Blutbahnen, deren Inhalt selbst nur unter einem ganz minimalen Drucke steht, schon durch einen geringen Druck von aussen her leicht einer Compression unterliegen, was Stauungen in den peripherwärts von ihnen gelegenen Gefässverzw eigungen zur Folge haben muss -, ferner einseitige Mydriais, ansgesprochenermaassen auf die Schläfenoder Hinterhauptsgegend localisirte Percussionsempfindlichkeit, Störungen in einzelnen Nervengebieten u. s. w. Diese localisirten Drucksymptome sind mehr dem parencbymatösen Abseess eigen, wiewohl sie auch beim interstitiellen in gewissem Grade vorkommen können; denn genau so wie der erstere ist auch der Inhalt des letzteren wie ein Fremdkörper aus der organischen Verbindung mit seiner Umgebung gelöst, doch wird hier die locale Druekwirkung abgesehwäeht dadurch! dass der Abscesseiter durch den allgemein erhöhten Druck, der von ihm ausgeht, gewissermaassen mehr in der Schwebe gehalten wird. Der locale Druck, den der interstitielle Abscess auf benachbarte Centra oder Leitungsbahnen ausübt, verhält sich zu dem, den der parenchymatöse austibt, etwa so wie der Druck einer Bleikugel auf ihre Unterlage in einem mit Wasser gefüllten Gefäss zu dem Druck einer gleichen Kugel in einem gleich grossen und mit der gleichen Menge des viel leichteren Alkohols gefullten Gefäss.

Besonders hervorheben möchte ich das Verhalten des $\mathrm{Pulses}$ bei den versehiedenen Druckverhältnissen im Gehirn. 
Die allgemeine Erhöhung des intracraniellen Druckes fuhrt zur Verlangsamung des Pulses, während ungleichmässige Vertheilung des Hirndrucks, einseitige Druckerhöhung, wenn sie einseitig das centrale Gebiet des recbten oder des linken N. vagus beeinflusst, Unregelmässigkeit des Pulses zur Folge hat. Da aber weder allgemeine noch den Vagus beeinflussende locale Druckerhöhung im Schädelinnern mit einem Hirnabscess nothwendiger Weise verbunden zu sein braucht, so ist weder Pulsverlangsamung noch Unregelmässigkeit des Pulses eine unerlässliche Begleiterscheinung eines jeden Hirnabscesses. Wo aber Druckbeeinflussung des Pulses zu finden ist, dort dürfte Pulsverlangsamung mehr auf einen interstitiellen, unregelmässiger Puls dagegen mehr auf einen parenchymatösen Abscess hinweisen - vorausgesetzt, dass die anderen Symptome auf einen Hirnabscess als den Ausgangspunkt der krankhaften Erseheinungen schliessen lassen. 1)

Schliesslich ist hinsichtlich der Symptome im Allgemeinen bei beiden Abscessarten noch hervorzuheben, dass der parenchymatöse Abscess mehr Herdsymptome, mehr Aasfallserscheinungen aufweist als der interstitielle, der mehr allgemeine Erscheinungen zur Folge hat; denn bei ersterem kommen zu den etwaigen localen Drucksymptomen, die ja auch beim interstitiellen Abscess, wenn schon, wie oben erwähnt, nur in geringerem Maasse zu finden sind, noch diejenigen Herd- oder Ausfallserseheinungen hinzu, die als directe Folge der Eins chmelzung der entsprechenden Centra oder gewisser leitender Verbindungsbahnen anzusehen sind.

Hierin ist aber ein weiterer Unterschied zwischen beiden Abscessarten begründet, nämlich hinsichtlich des Werthes und der Prognose ihrer operativen Entleerung in functioneller Beziehung. Hat ein parenchymatöser Abscess durch Einschmelzung gewisser Hirnabsebnitte zum Ausfall der von diesen ausgehenden Functionen geführt, so wird auch die operative

1) Einseitige Verschiebung der intracraniellen Druckverhältnisse wird auch durch extradurale Eiteransammlungen herbeigeführt, und deshalb ist unregelmässiger Puls dort, wo nach den ubrigen Erscheinungen ein Hirnabscess nicht vorliegt, ein wichtiger Fingerzeig dafür, dass ein extraduraler Abscess vorhanden sein dürfte. Ich bin wiederholt lediglich auf den unregelmässigen Puls hin bei Mastoidoperationen mit Absicht bis zur Dura, bezw. bis zum Sinus vorgedrungen und habe dann in der That auch mehrfach dort, zwischen Dura, bezw. Sinus und Schădelkapsel, Eiteransammlungen gefunden, auf die ausser dem unregelmăssigen Pulse vorher nichts hingewiesen hatte. 
Entleerung des Abscessinhaltes diese Functionen nicht wieder herzustellen im Stande sein; hat dagegen ein interstitieller Abscess durch Druck auf Centra oder Nervenbahnen in seiner Nachbarschaft beim Auseinanderdrängen der Hirnsubstanz ebenfalls zu Ausfallserscheinungen gefuhrt, so wird nach Entleerung des Abscesses auch eine allmälige Rückkehr der betreffenden Functionen zu erwarten sein, wenn nicht etwa bereits gänzliche Druckatrophie eingetreten ist. Ich sage ausdrticklich, eine „allmälige" Ruickkehr. Einmal missen die betreffenden Hirnabschnitte sich zunächst erst, wenn ich so sagen darf, wieder erholen; zum anderen ist aber mit der Entleerung des Abscesses noch nicht jegliche Druckwirkung auf seine Umgebung versohwunden, da wir ja bei unserem Verfahren, die dauernde Absaugung des Eiters durch Gazestreifen zu bewirken, die Abscesshöhle zunächst wieder tamponiren und dadurch erneut einen gewissen Druck verursachen. Hierin dïrfte auch, zum Theil wenigstens, die Erklärung dafür liegen, dass die Staunngserscheinungen am Augenhintergrunde oft noch wochenlang nach der operativen Entleerung des Abscesses zu beobachten sind.

Aber nicht nur für die functionelle, auch fur die vitale Prognose besteht ein Unterschied zwischen beiden Abscessarten. Der parenchymatöse Abscess hat, sofern er membranlos bezw. ohne Kapsel ist, auch nach der Entleerung noch die Neigung, sich durch das Weitergehen der Zerstörung an seinen Wandungen zu vergrössern; möglich, dass hier unter Umständen auch das oben angedeutete Activwerden etwaiger in dem Abscess neben anderen - vorhandener aërober Mikroorganismen mit dem durch die Abscesseröffnung bewirkten freien Luftzutritt eine Rolle spielt. Dem interstitiellen Abscess dagegen ist, namentlich, wenn er bereits eine feste Balgkapsel besitzt, mit seiner Entleerung der Anlass, sich noch weiter auszubreiten, genommen, da, selbst wenn von seinen Wandungen her noch eine Zeitlang eine Einwanderung von neuen Eiterkörperchen in die Abscesshöble stattfindet, diese durch die drainirenden Gazestreifen nach aussen befördert werden. Im Allgemeinen würde also der interstitielle Abscess prognostisch quoad functiones und quoad vitam, gùnstiger sein als der parenchymatöse.

Betrachten wir nun an der Hand dieses kurz skizzirten Sehemas, dem sich übrigens theoretisch noch weitere untersehiedliche Gesichtspunkte hinzuconstruiren liessen, unsere oben geschilderten vier Fälle, so finden wir - abgesehen von Fall I, der nur 
ungenügend beobachtet war, - dass nur Fall III sich rein in das eine der von uns aufgestellten Schemata einpassen lässt.

Fall I ist im Hinblick auf den Obductionsbefund mit Sicherheit als parenchymatöser Abseess anzusprechen, ist aber eben nicht gentigend beobachtet, um alle die geschilderten Gesichtspunkte an ihm prüfen zu können. Jedenfalls dürfte das Fehlen allgemeiner Drucksymptome nur aus der parenchymatösen Eigenschaft des Abscesses zu erklären sein. Ein interstitieller Abscess von auch nur annähernd derselben Grösse würde zweifellos in die Augen springende Hirndruck symptome zur Folge gehabthaben und wäre dann auch gewiss intra vitam diagnosticirt worden.

Fall II war nach dem Obductionsbefund ohne $\mathrm{Z}$ weifel ebenfalls ein parenchymatöser Abscess. Damit stimmt der Gestank und der bakteriologisehe Befund des Abscesseiters (Kokken gleichzeitig mit Stäbchen, von denen eines eine Art von Bac. coli darstellte), das Fehlen einer Membran und, wenn man will, auch der tödtliche Ausgang. Und doch waren hier allgemeine Hirndrucksymptome vorhanden; zwar fehlten Staungserscheinungen am Augenhintergrunde, aber Brechneigung und verlangsamter Puls standen im Vordergrunde der Symptome, und namentlich letzterer war fur die sehnelle Sicherstellung der Diagnose von grossem Werth.

Fall III war e in paren chymatöser Abscess. Diejauchige, stinkende Beschaffenheit des Abscessinhalts, der nur spärliche Eiterkörperchen, dafür aber reichliche Detritusmassen und verschiedene Mikroorganismen - mehrere Strepto- und DiplokokkenArten, von letzteren einzelne mit Hof, sowie kurze Stäbehen (Proteus) - enthielt, ferner rechtsseitige Mydriasis sowie nur rechtsseitige Stauungserscheinungen am Augenhintergrunde, der nicht verlangsamte Puls, das ichorrhämische Fieber und die septische Endocarditis und schliesslich das Fehlen einer Membran (wenigstens ist eine solche nicht erwähnt) lassen diesen Fall zwanglos in unser Schema des parenchymatösen Abscesses einftugen. Den tödtlichen Ausgang als eine weitere Stütze für unsere Annahme des parenchymatösen Charakters anzusprechen, ist mit Rücksicht anf den zweiten, nicht eröffneten Abscess nicht angängig.

Fall IV war ein interstitieller Abscess. Die Grinde, welche nach unseren obigen Ausführungen hierfür sprechen, sind: Der günstige Ausgang in vollständige Heilung ohne Hinterlassung jeglicher Ausfallserscheinung, der nicht stinkende, reine Eiter, der nur Streptokokken in Reincultur enthielt, der geringe Grad. 
der Fiebererscheinungen und schliesslich, als Ausdruck des erhöhten allgemeinen Hirndrucks, 4 Punkte, nämlich die allgemeinen Kopfschmerzen, die subjectiv die einzigen Klagen der Kranken bildeten, dann das häufige und langanhaltende Erbrechen, wodurch die Ernährung durch Clysmata für lange Zeit nothwendig wurde, ferner die Beiderseitigkeit der Stauungserscheinungen am Augenhintergrunde, wenn diese anch links intensiver waren als rechts (dies wirde mit einem neben der allgemeinen Druckerhöhung vorhandenen localem Drucke des Abscesses auf die zahlreichen Blutleiter in der hinteren Schädelgrube und ihrer Nachbarschaft leicht zu erklären sein), und viertens das Deliriren und Phantasiren und aufgeregte Wesen der Kranken. Eines aber fehlt zur Vollständigkeit des Bildes, nämlich die Verlangsamung des Pulses.

Wenn so das Krankheitsbild bei Hirnabscessen nicht immer - ja wir können weiter gehen und sagen: wohl nur in der Minderzahl der Fälle - genau in allen Punkten dem Schema der einen oder der anderen Abscessart entspricht, so liegt hierin kein Beweis gegen die Richtigkeit der grundsätzlichen Unterscheidung der beiden Abscessarten. Es kann sehr leicht bei einem Anfangs rein parenchymatösen Abseess durch den Reiz, den dieser auf seine Umgebung ausubt, oder durch das nachträgliche Eindringen der entsprechenden Mikroorganismen zu einer entzündlichen Einwanderung von Eiterkörperchen aus der bindegewebigen Umgebung in den Jaucheherd hinein kommen; dann haben wir einen destructiven und einen entzündlich productiven Vorgang gleichzeitig und damit eine $\mathrm{M}$ is chform des Abscesses vor uns. Desgleichen kann es bei einem zunächst rein interstitiellen Abscess durch Druck auf die umgebende Hirnsubstanz oder durch nachträgliches Eindringen von Fänlnissbakterien zu Nekrose und Zerfall des benachbarten Hirngewebes kommen; auch dann liegt eine Combination von interstitiellem und parenchymatösem Abscess vor. Dass dann die Symptome dem combinirten Wesen des zu Grunde liegenden pathologisch-anatonischen Zustandes entsprechen werden, bedarf keines Beweises. Es ist nicht ausftihrbar, alle auf diese Art möglichen Combinationen im Einzelnen durehzusprechen, aber es liegt auf der Hand, dass so die allerverschiedensten Krankheitsbilder zu Stande kommen können.

Die Mis ch form scheint, soweit meine eigenen Beobachtungen und ein Ueberblick über die Litteratur mir einen Schluss ge- 
statten, die häufigere zu sein. Jedenfalls kann man bei allen Abscessen, die einigermaassen grössere Dimensionen erreicht laben, annehmen, dass sie nicht mehr die reine Form des parenchymatösen oder des interstitiellen Abscesses, sondern eine Mischform beider Arten darstellen. Im Beginn ihrer Entwicklung aber gehören wohl die meisten Hirnabscesse eine Zeit lang rein dem einen oder dem anderen Typus an, wenn auch die Möglichkeit eines gleichzeitigen Eindringens von Eiter- bezw. Entzündungserregern zusammen mit Fäulnisserregern und damit das gleichzeitige Einsetzen entzïndlich-productiver und destructiver Vorgänge im Gehirn nicht geleugnet werden soll.

Meine Unterscheidung zwischen den beiden Abscessarten baut sich nicht auf blossen theoretischen Erwägungen auf, sondern sie entspringt eingehenden klinischen Beobachtungen, die auf andere Weise nicht zu erklären sind. In hohem Grade erwïnscht wäre es natürlich, wenn es gelänge, diese durch klinische Erfahrungen und Beobachtungen am Krankenbett gewonnenen Resultate nun in Zukunft auch durch die pathologisch-anatomische Untersuchung zu bestätigen. Jeder Gehirnabscess, der auf den Sectionstisch kommt, sollte nicht nur: makroskopiseh, sondern auch mikroskopisch-histologiseh auf das Genaueste untersucht werden.

Kurz möchte ich noch - ausserhalb des Rahmens der geschilderten Untersoheidung der beiden Abscessarten - anf eine Erscheinung hinweisen, die leicht zu einer Verwechselung des Hirnabscesses mit Meningitis führen kann; es ist dies eine bei Hirnabscessen von uns nicht selten beobachtete - z. B. unsere Fälle 2, 3 und 4 - steife Haltung des Kopfes, die nicht die Folge einer wirklichen Nackenstarre ist. Gewiss kommt echte Nackensteifigkeit bei Hirnabscessen in seltenen Fällen vor; dann ist sie wohl immer die Folge einer den Abscess complicirenden Meningitis oder meningitischen Reizung. Dagegen besteht die Erscheinung, die ich hier meine, darin, dass der Kranke, da seine Kopfschmerzen durch jede, auch noch so leichte Bewegung des Kopfes gesteigert werden, um dies zu vermeiden, den Kopf mit Absicht unbewegt hält und auch dem Versuche, ihn passir zu bewegen, Widerstand entgegensetzt. Besonders charakteristisch ist diese Kopfhaltung, wie mein hochverehrter früberer Chef, Herr Geheimrath Trautmann immer hervorhebt, wenn der Kranke noch im Stande ist zu gehen; der Kopf wird alsdann etwas nach hintentuber gebeugt und ohne 
jede Bewegung still gehalten; hierzu kommt dann noch ein langsamer, vorsichtiger, gespreizter $\mathrm{Gang}$, der ebenfalls der Absicht, Kopferschtitterungen za vermeiden, entspringt. Manche Kranke unterstutzen dies noch dadurch, dass sie sich mit einer oder gar mit beiden Händen den Nacken oder den Hinterkopf balten. Ein so einhergehender Kranker bietet ein überaus charakteristisches Bild, das die Stellung der Diagnose recht erleichtern kann. Bei bettlägerigen Kranken liegt aber umgekehrt hier die Gefahr einer Verwechselung mit wirklicher Nackensteifigkeit vor; so war z. B. in unserem Falle IV hauptsächlich auf dieses Symptom hin Anfangs von anderer Seite die Diagnose auf Meningitis gestellt worden.

Zum Schlusse noch ein Wort zur Technik der Operation des Hirnabscesses. Wir haben bis jetzt grundsätzlich daran festgehalten, den Gehirnabscess von aussen her, durch die Schläfenschuppe bezw. durch das Hinterhaupt hindurch $\mathrm{zn}$ entleeren. Die Grtinde hierfür habe ich an anderer Stellet) ausfuhrlicher dargelegt. Auch unsere neueren Erfahrungen sind nicht geeignet, uns von diesem Standpunkte abzubringen. Allerdings könnte der Misserfolg in unserem Falle III, wo die Absangung des Eiters durch die Hinterhauptwunde hindurch nicht genitgend war, als ein Beweis daftir in Anspruch genommen werden, dass es doch besser sei, von der Mastoidoperationswunde her zu operiren. In der That würde in diesem Falle die Anlegung, wenn ich so sagen darf, einer Gegenöffnung von der Mastoidwunde her von guter Wirkung gewesen sein. Doch sollte man sich zu diesem Vorgehen - Anlegung einer Gegenöffnung nur dann entschliessen, wenn das absolute Fehlen von Erscheinungen erhöhten Hirndrucks einigermassen die Gewähr bietet, dass ein grösserer Hirnprolaps in die Warzenfortsatzwundhöhle binein nicht zu befürchten steht. Ist diese Bedingung schon von vornherein erfullt, so würde auch dagegen, den Abscess a priori von der Mastoidwundhöhle her zu eröffnen, grundsätzlich nichts einzuwenden sein.

1) "Zur operativen Behandlung der otitischen Hirnhautentzündungen" am Schlusse. Deutsche med. Wochenschr. 1899. Nr. 45. 